\title{
Reconstruction of the First Metacarpal after Giant Cell Tumor Excision using Non-Vascularised Fibular Autograft, Ligament Reconstruction and Tendon Interposition Arthroplasty of the Carpometacarpal joint: A Case Report
}

\author{
Nathaniel S. Orillaza, Jr., ${ }^{1}$ Tammy L. dela Rosa ${ }^{1}$ and \\ ASTRO (Advanced Study and Research in Orthopedics) Study Group ${ }^{2}$ \\ ${ }^{1}$ Section of Hand and Micro Surgery, Department of Orthopedics, College of Medicine and Philippine General Hospital, \\ University of the Philippines Manila \\ ${ }^{2}$ National Institutes of Health, University of the Philippines Manila
}

\begin{abstract}
We report an innovative approach to reconstructing the defect after excision of a giant cell tumor (GCT) involving the entire first metacarpal. Reconstruction was performed using nonvascularized fibular autograft fused at the metacarpo-phalangeal (MP) joint and soft tissue arthroplasty using ligament reconstruction and tendon interposition (LRTI) at the carpometacarpal (CMC) joint of the thumb.

Two years after the procedure, the CMC joint had functional pain-free motion and radiographs showed fusion of the MP joint. The patient did not present with any major complication and was satisfied with the outcome of the procedure.
\end{abstract}

Key Words: metacarpal giant cell tumor, suspension arthroplasty, hand tumor reconstruction, non-vascularized fibula graft

\section{Introduction}

Giant cell tumor (GCT) of the bone is a benign but locally aggressive lesion. It is a rare tumor composed of connective tissue stromal cells having the capacity to recruit and interact with multinucleated giant cells that exhibit the phenotypic features of osteoclasts. ${ }^{1}$ In the small bones, they are often juxta-articular and break through the surrounding cortex at the time of diagnosis. It is rarely found in the hand, with an incidence of around $2-5 \%$ of all GCTs. ${ }^{1,2}$

There have been very few reports on managing GCTs of small bones, much less for the thumb metacarpal. Cases involving a large segment of the bone extending to the proximal or distal ends usually end with fused joints on both ends after excision of the affected bone. This is not a problem for the metacarpo-phalangeal (MP) and the interphalangeal (IP) joints. Preserving motion on the carpo-metacarpal

Corresponding author: Nathaniel S. Orillaza, Jr., MD

Section of Hand and Micro Surgery, Department of Orthopedics Philippine General Hospital

University of the Philippines Manila

Taft Avenue, Ermita, Manila 1000 Philippines

Telephone: +632 5548466

Email: docnats@yahoo.com
(CMC), however, where most of the thumb movement comes from, is optimal in retaining as much function as possible.

Ligament reconstruction and tendon interposition of the first CMC joint was originally described by Eaton and Littler. $^{3}$ This was designed to provide stable, pain-free motion for basal arthritis of the thumb. It is generally accepted as the preferred treatment for first CMC joint arthritis. ${ }^{4}$ No such procedure has been reported for preserving motion in reconstructing the defect after tumor excision.

\section{Case Report}

We present the case of a 24-year-old, female, righthanded domestic helper, who consulted for a 4-week history of rapidly enlarging mass on the dorsum of the thumb metacarpal area of the dominant hand (Figure 1). She experienced pain and had limited motion at the CMC and MP joints of the thumb. Using Disability of Arm, Shoulder and Hand (DASH), ${ }^{5}$ she presented with $49 \%$ disability of the affected hand.

Radiographs showed a large, expansile lytic lesion involving the entire first metacarpal. The tumor had no matrix and had thinned out the cortices (Figure 2). A core needle biopsy was performed, revealing giant cell tumor of the bone.

Surgery was performed with the aim of performing wide excision and reconstruction that would retain as much function as possible. The incision was made over the dorsal aspect of the thumb to include the puncture site of the biopsy. The thenar muscles on the ulnar and palmar sides and the fascia with a skin island around the biopsy site dorsally served as the margins. The proximal aspect was excised with the trapezium and distally was disarticulated at the MP joint. The excision included the opponens, abductor and short flexor of the thumb. The abductor pollicis longus was detached at the attachment at the base of the metacarpal. The extensor policis longus was also transected because it passed through the tumor but was repaired after 
excision of the tumor. The volar structures including tendons and neurovascular bundles were preserved.

After excision of the tumor measuring $6 \times 5 \times 3 \mathrm{~cm}$, (Figure 3a) the margins showed no gross contamination. There was good capillary refill of the thumb and the skin flaps were viable (Figure 3b).

A $5.5 \mathrm{~cm}$ non-vascularized fibular autograft was harvested from the contralateral leg.

The distal autograft was fused with the proximal phalanx base using tension band wiring. The CMC joint was reconstructed with soft tissue arthroplasty using the flexor carpi radialis (FCR). The FCR was cut as far proximal as possible at the musculotendinous junction and delivered distally at the level of the first CMC joint (Figure 4). It was inserted through a hole at the proximal end of the autograft and tied and sutured to itself to create a suspension ligament. The remaining tendon was folded and sutured to an "anchovy" which was interposed between the autograft and the space left with the excision of the trapezium. (Figure 5)

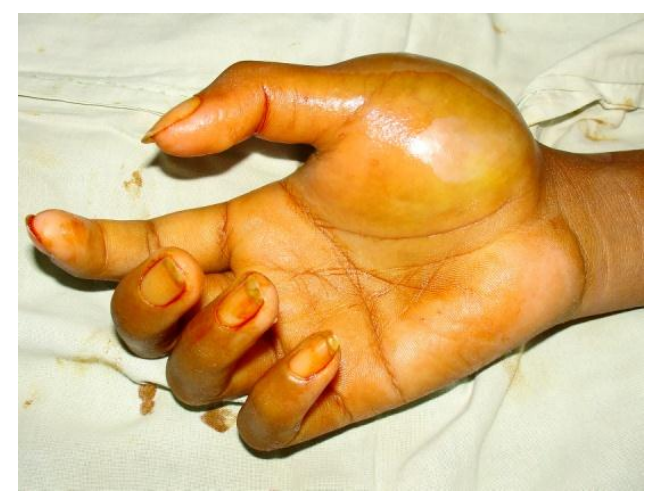

Figure 1. Large tumor, dorsal side of thumb metacarpal area.

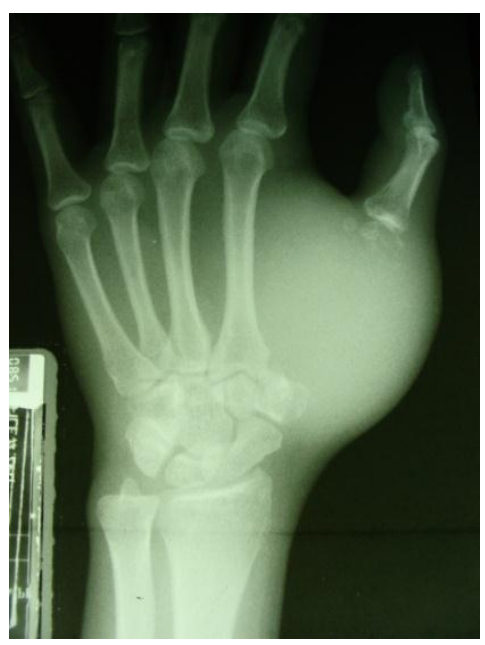

Figure 2. On radiographic PA view of the hand: expansile, lytic lesion involving the entire thumb metacarpal.

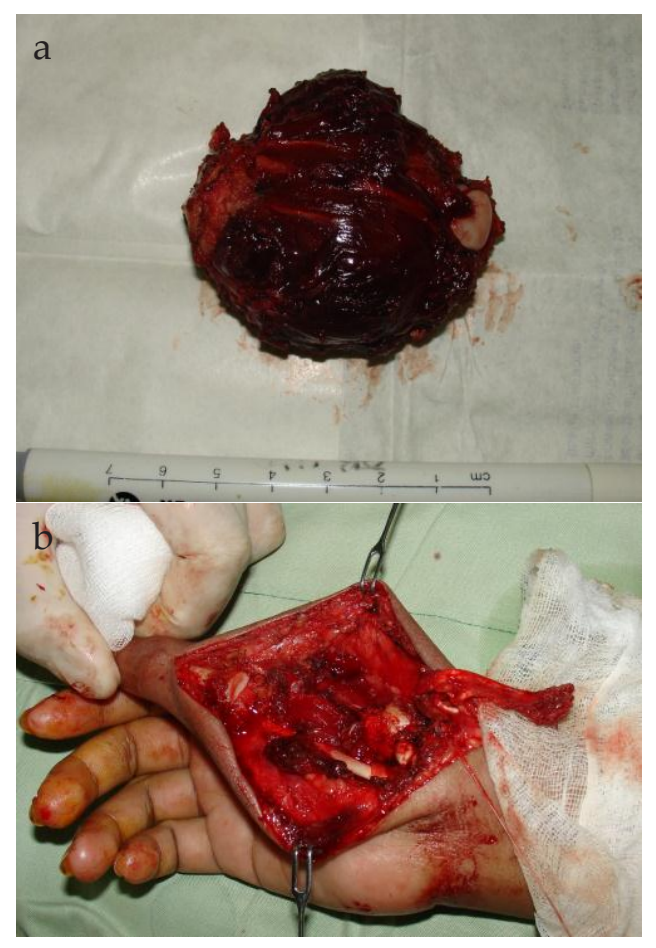

Figure 3. (a) The tumor excised with the MP joint, trapezium and thenar muscles as margins of excision, (b) the defect after excision with transected EPL.

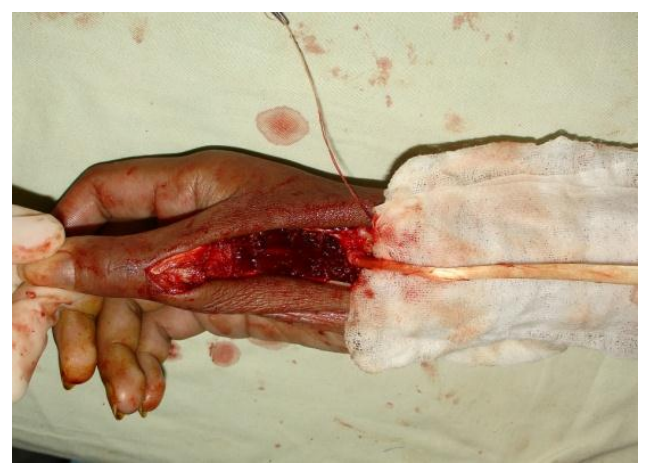

Figure 4. The FCR transected proximally and delivered to thumb CMC joint.

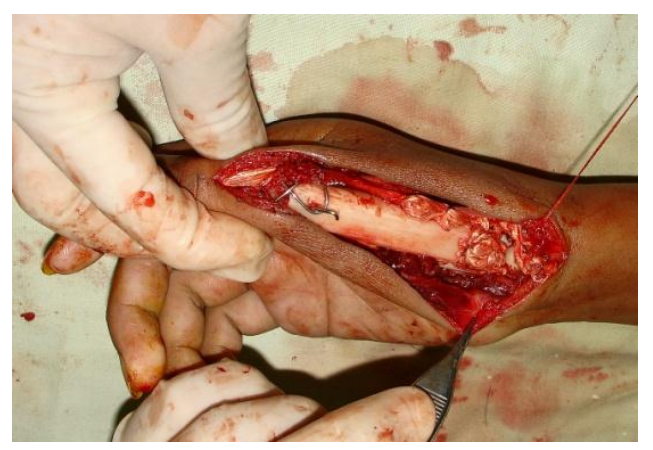

Figure 5. Thumb MP joint fused with tension band wire and CMC joint reconstructed with LRTI using the FCR. 
The autograft was initially stabilized with a transmetacarpal pin and the hand was immobilized with a thumb spica.

The wounds healed well after surgery. The transmetacarpal pin was removed 6 weeks postoperatively and the thumb spica 8 weeks postoperatively followed by assisted active range of motion exercises.

\section{Results}

\section{Early Outcome}

Pain-free, active abduction-adduction limited to 15 degrees of motion was observed at 12 weeks postoperatively. Monthly follow-ups showed gradual increase in range with no signs of infection, recurrence and metastasis to the lungs.

At 6 months postoperatively, pain-free active composite flexion-extension of up to $40^{\circ}$ arc of motion with grip strength of $4 / 5$. Radiographs showed a fused MP and a floating CMC. (Figure 6)

She has been able to perform her duties as a domestic helper with certain limitations in performing procedures requiring a tight grip and heavy lifting using the affected hand.

\section{Long-term Outcome}

At 2 years post-surgery, there was minimal increase in range of motion to $45^{\circ}$ arc of composite flexion-extension and adduction-abduction, but the thumb maintained pain-free motion (Figure 7). No oncologic complications were observed on latest follow-up. Radiographs showed maintained fusion with no implant failure.

The patient presented with good function and is satisfied with her condition as measured by pre- and postoperative $\mathrm{DASH}^{5}$ and serial postoperative Revised Musculoskeletal Tumor Society Rating Scale (MSTS) ${ }^{6}$ at 6 and 24 months follow-up (Tables 1 and 2).

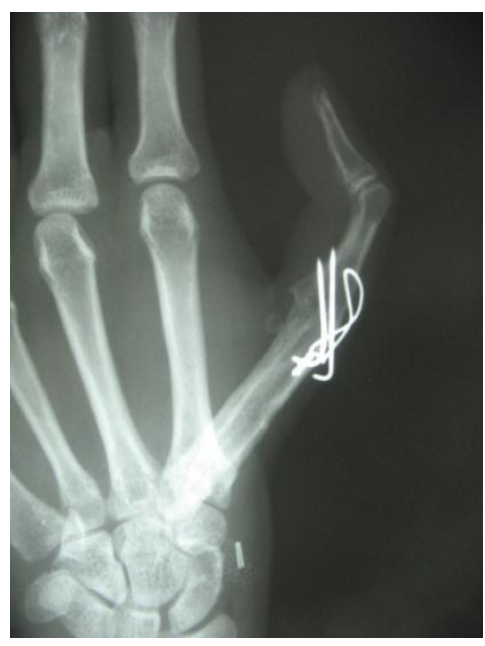

Figure 6. On follow-up radiographs, thumb MP joint fused at 6 months after surgery.

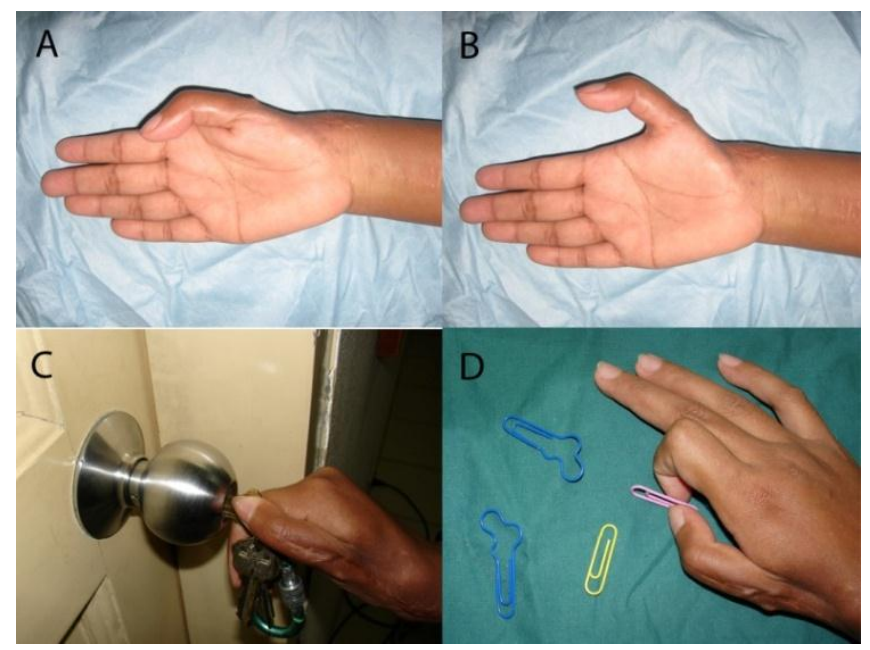

Figure 7. Clinical pictures at 2 years after surgery. (A/B) Wounds healed well with limited motion at CMC joint, (C/D) functional fine motor movements.

Table 1. Musculoskeletal Tumor Society (MSTS) Rating at 6 months and 2 years after surgery

\begin{tabular}{|c|c|c|c|c|c|c|c|}
\hline \multicolumn{8}{|c|}{ MSTC Scores } \\
\hline & 兊 & 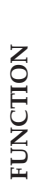 & 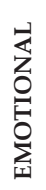 & 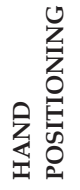 & 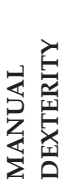 & 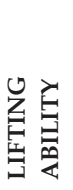 & $\begin{array}{l}\underbrace{a}_{0} \\
e \\
0 \\
0 \\
0\end{array}$ \\
\hline $\begin{array}{l}6 \\
\text { mos } \\
\text { post }\end{array}$ & $5 / 5$ & $4 / 5$ & $5 / 5$ & $5 / 5$ & $4 / 5$ & $3 / 5$ & $\begin{array}{l}26 / 30 \\
86 \%\end{array}$ \\
\hline $\begin{array}{l}2 \text { yrs } \\
\text { post }\end{array}$ & $5 / 5$ & $4 / 5$ & $5 / 5$ & $5 / 5$ & $4 / 5$ & $4 / 5$ & $\begin{array}{l}27 / 30 \\
90 \%\end{array}$ \\
\hline
\end{tabular}

Table 2. Disabilities of Arm, Shoulder and Hand (DASH) at Pre-op, 6 months and 2 years after surgery

\begin{tabular}{lll}
\hline & \multicolumn{1}{c}{ DASH Scores } \\
Pre-op & 6 mos post & 2 years post \\
\hline 59 & 28 & 28 \\
$49 \%$ disability & $23 \%$ disability & $23 \%$ disability \\
\hline
\end{tabular}

\section{Discussion}

Managing aggressive tumors of the bones of the hand entails critical decision making with the purpose of balancing the oncologic and functional outcomes. Recent advancements in tumor management have expanded the capacity for limb conservation increasing the demand for creative and innovative techniques for preserving function without sacrificing risk for recurrence and other complications. Such procedures also pose the need for immediate stabilization to optimize the advantages of early motion and to prevent stiffness. 
Several treatment options have been presented with the same goal of eradicating the tumor while maintaining function. Wide surgical excision is preferred but it entails sacrificing normal tissue such as muscles and neurovascular structures around the tumor which in turn can significantly diminish function. Curettage and bone grafting with or without adjuvant modalities are acceptable procedures for less aggressive lesions. However, even with adjuvant modalities, it is impossible to completely remove the entire tumor with this procedure. In the hand, curettage and grafting resulted in as high as $80 \%$ recurrence. ${ }^{2}$

There have been very few reports on reconstructive options after excision of tumors of the first metacarpal. Previously published case reports involved the use of metatarsal head grafts and metal prosthesis to retain motion. ${ }^{78}$ For low-demand patients, the preferred option was fusion with autograft after excision of the metacarpal. This provides early stability but greatly limits motion. ${ }^{9}$ We presented a combination of autograft reconstruction, MP fusion and soft-tissue arthroplasty of the CMC joint of the thumb.

Unlike the classic LRTI for arthritis, additional challenges encountered in our procedure include the use of a non-vascularized autograft instead of the metacarpal, loss of intrinsic musculature around the thumb with the excision procedure and fused MP joint. All these conditions would expectedly present with relatively less postoperative function of the thumb. However, this did not necessarily equate with unsatisfactory results. The procedure allowed adequate tumor excision while retaining key structures to regain motion. Rigid MP fixation allowed early motion resulting in early return to function. Our patient has been able to perform all activities of daily living without assistance even with limited over-all motion. She has been able to perform her duties at work with minimal discomfort and was satisfied as reflected by her DASH and MSTS scores.

\section{Summary}

We presented an innovative reconstructive technique after excision of a giant cell tumor involving the entire first metacarpal. The defect was replaced with a nonvascularized fibula and motion was preserved at the CMC joint using ligament reconstruction tendon interposition arthroplasty. Results at 2 years show good function and patient satisfaction with no major complications.

\section{References}

1. Goldenberg RR, Campbell CJ, Bonfiglio M. Giant cell tumor of bone. An analysis of 218 cases. J Bone Joint Surg (Am). 1970; 52(4):619-64.

2. Averill RM, Smith RJ, Campbell CJ. Giant cell tumors of the bones of the hand. J Hand Surg Am. 1980; 5(1):39-50.

3. Eaton RG, Littler JW. Ligament reconstruction for the painful thumb carpometacarpal joint. J Bone Joint Surg Am. 1973; 55(8):1655-66.

4. Ghavami A, Oishi SN. Thumb trapeziometacarpal arthritis: treatment with ligament reconstruction tendon interposition arthroplasty. Plast Reconstr Surg. 2006; 117(6):116e-128e.

5. Hudak PL, Amadio PC, Bombardier C. Development of an upper extremity outcome measure: the DASH (disabilities of the arm, shoulder and hand) [corrected]. The Upper Extremity Collaborative Group (UECG). Am J Ind Med. 1996; 29(6):602-8.

6. Enneking WF, Dunham W, Gebhardt MC, Malawar M, Pritchard DJ. A system for the functional evaluation of reconstructive procedures after surgical treatment of tumors of the musculoskeletal system. Clin Orthop Relat Res. 1993; (286):241-6.

7. Kettelkamp DB, Ramsey P. Experimental and clinical autogenous distal metacarpal reconstruction. Clin Orthop Relat Res. 1971; 74:129-37.

8. Dingels WR, Rolle HJ. Case report of a giant cell tumor of the second metacarpal bone and implantation of a cement prosthesis. Handchirurgie. 1979; 11(3-4):251-4.

9. Jain S, Yadav C, Rastogi S, Msoi HN, Kapoor SK. Large giant cell tumor of first metacarpal: a management dilemma. Curr Orthop Pract. 2010; 21(6):624-7. 